\title{
Elastic electron scattering by halocarbon radicals in the independent atom model approach ${ }^{\star}$
}

\author{
Sándor Demes ${ }^{1, a, b}$, Vladimir Kelemen ${ }^{2}$, and Eugene Remeta ${ }^{2}$ \\ 1 Institute for Nuclear Research (ATOMKI), Bem tér 18/c, 4026 Debrecen, Hungary \\ 2 Institute of Electron Physics, National Academy of Sciences of Ukraine, Universitetska str. 21, 88017 Uzhhorod, Ukraine
}

Received 25 October 2019 / Received in final form 1 February 2020

Published online 19 March 2020

(c) The Author(s) 2020. This article is published with open access at Springerlink.com

\begin{abstract}
In order to study the elastic scattering of electrons by $\mathrm{CF}_{n}(n=1-4)$ molecular targets the independent atom model (IAM) is used with the optical potential (OP) method. The scattering cross sections were calculated in two approximations of the model - the IAM approach is used for the differential, while the Additivity Rule (IAM-AR) is used for the integral cross sections. The amplitudes of electron scattering by the carbon and fluorine atoms of the target molecules are calculated from the corresponding phase shifts, using the real and complex optical potential method. The parameter-free real part of the OP is calculated from the corresponding atomic characteristics - nuclear charge, electron density and static dipole polarizability. The differential and integral cross sections are calculated at equilibrium internuclear distances of the $\mathrm{CF}_{n}$ molecules. They were compared with the available experimental data and with other theoretical results. A good overall agreement was observed while comparing our integral cross sections with the measured data. The level of the agreement however strongly depends on the target molecule, and a good consistency is observed typically above certain collision energies: from $10 \mathrm{eV}$ in case of $\mathrm{CF}_{2}$, above $15-20 \mathrm{eV}$ for $\mathrm{CF}_{3}$ and from $40 \mathrm{eV}$ in case of $\mathrm{CF}_{4}$. Similar tendencies were found in case of the differential cross sections for a wide range of scattering angles at collision energies above $10 \mathrm{eV}$ in case of $\mathrm{CF}_{2}$, above 15-20 eV for $\mathrm{CF}_{3}$, while in case of $\mathrm{CF}_{4}-$ above $20 \mathrm{eV}$.
\end{abstract}

\section{Introduction}

The physical electronics is a wide scientific research area, and its achievements could be applied in several state-ofthe-art technologies: from low-temperature plasma, semiconductor production and material science up to light industry and environmental protection [1]. For example, the plasma discharge could be treated as a medium, where a high number of electron collisions take place with atoms and/or molecules. Electron collisions with molecules play a very important role in several scientific and applied fields: from the investigation of the ionizing radiation effects on the human body and the DNA up to the kinetic modelling of plasma environments, including those of the

* Contribution to the Topical Issue "Low-Energy Positron and Positronium Physics and Electron-Molecule Collisions and Swarms (POSMOL 2019)", edited by Michael Brunger, David Cassidy, Saša Dujko, Dragana Marić, Joan Marler, James Sullivan, Juraj Fedor.

${ }^{\text {a }}$ e-mail: demes.sandor@atomki.mta.hu

${ }^{\mathrm{b}}$ Present address: Normandie University Le Havre, LOMCUMR 6294 CNRS, 53 rue de Prony, BP 540, 76058 Le Havre Cedex, France interstellar medium. Moreover, the electron interactions with atoms and molecules are the driving forces in lowtemperature plasma processes.

The absolute values of electron-molecule scattering cross sections play a crucial role in the plasma reactor modelling as well as in the control of plasma processing efficiency of gas mixtures. However, there are only limited data about the low-energy collisions in these gases. Therefore, different scattering approaches should be used in order to obtain relevant knowledge about the collisions, e.g. on the energy and angular dependencies of the processes. These type of theoretical predictions can be used for modelling of complex processes, which are not easy to handle experimentally (for example, due to the toxic or highly reactive species).

The fluorine-containing radicals are the key components of the different gas-discharge and low-energy plasma environments (for more details see the numerous experimental and theoretical works [1-13]). Among the traditional feedstock gases in plasma-assisted semiconductor production the following molecular gases could be mentioned: $\mathrm{CF}_{4}, \mathrm{C}_{2} \mathrm{~F}_{6}, \mathrm{C}_{3} \mathrm{~F}_{8}, \mathrm{c}-\mathrm{C}_{4} \mathrm{~F}_{8}$ and $\mathrm{GeF}_{4}, \mathrm{SiF}_{4}$. In the plasma these molecules undergo to fragmentation processes due to inelastic collisions, which lead to the production of ionized and neutral radicals, including $\mathrm{CF}, \mathrm{CF}_{2}$ and $\mathrm{CF}_{3}$. 
It is worth noting that similar molecules could be also important in those processes, in which the carbon and fluorine atoms are exchanged with $\mathrm{Si}$ and $\mathrm{Ge}$ or $\mathrm{Cl}, \mathrm{Br}$ and I, respectively $[14,15]$. The relative ratio and the role of different radicals in the plasma processes are poorly understood at the moment. The fluorocarbon radicals have some common properties, e.g. large dipole moment and dipole polarizability and in most cases they have also openshell-type electronic structure. The large scattering cross sections amplitudes could be also related with these properties. Unfortunately, the experimental studies of the above mentioned radicals are rather limited, because of the complexity of preparation of stable fluorocarbon beams with appropriate particle density.

The name of a recent work [2] "Anomalously large low-energy elastic cross sections for electron scattering from the $\mathrm{CF}_{3}$ radical" primarily states its aim - how the large integral cross section of electron scattering could be explained below $15 \mathrm{eV}$ collision energies? The authors of reference [3], which is the continuation of paper [2], presented a series of experimental and theoretical cross sections for $e^{-}+\mathrm{CF}_{3}$ elastic scattering at collision energies between 7 and $50 \mathrm{eV}$. The target radicals were obtained by the authors by pyrolysis from $\mathrm{CF}_{3} \mathrm{I}$ molecules at $817^{\circ} \mathrm{C}$. However, in these processes other atomic and molecular fragments were produced as well, with the following relative concentrations: $\mathrm{CF}_{3}(23 \%), \mathrm{I}(33 \%), \mathrm{CF}_{3} \mathrm{I}(25 \%), \mathrm{I}_{2}$ $(7 \%)$ and $\mathrm{C}_{2} \mathrm{~F}_{6}(12 \%)$. The relative ratios of these fractions were also used in the determination of the absolute cross section values (see $[2,3]$ for more details). At the same time, the states of the particular radicals in these experiments are not well-identified. For example, if the $\mathrm{CF}_{3}$ and $\mathrm{CF}_{2}$ target radicals are produced in vibrationally excited states, it could lead to overestimated cross-section values, compared to the ground state.

It is worth noting here that the experimental cross sections in $[2,3]$ were compared with the results of several theoretical calculations. They applied the Schwinger multichannel method (SMC) in the simple static-exchange (SE) approximation as well as the independent atom model with screening corrections (IAM-SCAR), both with and without ground-state dipole corrections. Unfortunately, none of the theoretical data reproduce the behaviour of the integral cross sections (ICS) below $20 \mathrm{eV}$. They are also only in a qualitative agreement with the experiments in case of differential cross sections (DCS) at these energies. In the quantitative analysis they underestimate the measured data by an order of magnitude.

The independent atom model (IAM) is a relatively simple and widely used theoretical approach for studying the electron scattering dynamics by molecules. The model uses the interaction potentials, phase shifts and scattering amplitudes, which are calculated for electron scattering by the particular atoms of the molecule. So, the molecular target in the IAM framework is treated as a collection of atoms (without symmetry), which are located at welldefined distances from each other. The model was proposed by Mott and Massey [16], and it was intensively used in recent studies $[3,14,15,17]$ to calculate the differential and integral scattering cross sections. The IAMSCAR method is a novel version of the model, which takes into account the interatomic screening effects by a multiplicative, energy-dependent factor. This factor was derived both for total [18] and for differential cross sections [19], increasing the precision of the model at lower collision energies.

Nowadays more sophisticated methods are also available, which treat the interaction potentials and the scattering amplitudes in a more convenient manner, taking into account purely molecular properties. These models use, for example, a symmetry-adapted, single-centre expansion of the molecular wave function to calculate the electron densities. Such methods were proposed with spherical [20] and single-centre [21] potentials.

In the present work we propose a joint theoretical analysis for elastic electron scattering by the $\mathrm{CF}_{4}$ molecule and its $\mathrm{CF}_{n}(n=1-3)$ radicals, using two approximations of the well-known independent atom model. The method is based on quantum-mechanical electron-atom scattering amplitudes. To calculate the amplitudes, the real and the complex optical potential (OP) methods were used. The cross sections in this work are compared with the available experimental and theoretical data for $\mathrm{CF}_{n}$ systems.

\section{Theory}

\subsection{Scattering cross sections and amplitudes}

The scattering of an electron with momentum $k$ on an $N$-atomic molecule by angle $\theta$ could be characterized theoretically by the $F(\theta, k)$ (direct) and $G(\theta, k)$ (spinflip) scattering amplitudes. Within the independent atom model framework, they correspond to the sum of the particular atomic scattering amplitudes $f_{m}(\theta, k)$ and $g_{m}(\theta, k)$ (see for example $[1-3,5,6,9,16,17,22])$ :

$$
\begin{aligned}
& F(\theta, k)=\sum_{i}^{N} \exp \left(i \boldsymbol{k} \cdot \boldsymbol{r}_{i}\right) \cdot f_{i}(\theta, k), \\
& G(\theta, k)=\sum_{i}^{N} \exp \left(i \boldsymbol{k} \cdot \boldsymbol{r}_{i}\right) \cdot g_{i}(\theta, k) .
\end{aligned}
$$

For the differential cross section then we have:

$$
\begin{aligned}
\mathrm{d} \sigma_{\text {orient }} / \mathrm{d} \Omega=\mid & |F(\theta, k)|^{2}+|G(\theta, k)|^{2}=\sum_{i, j}^{N} \exp \left(i \boldsymbol{k} \cdot \boldsymbol{r}_{i j}\right) \\
& \times\left[f_{i}(\theta, k) \cdot f_{j}^{*}(\theta, k)+g_{i}(\theta, k) \cdot g_{j}^{*}(\theta, k)\right],
\end{aligned}
$$

where $\boldsymbol{r}_{i j}=\boldsymbol{r}_{i}-\boldsymbol{r}_{j}$ are the internuclear distances.

The OP method is used to study the behaviour of the differential as well as the integral elastic and momentum transfer cross sections of electron scattering by molecules [23-26]. In the IAM framework the DCS of elastic electron scattering by an $\mathrm{N}$-atomic molecule, after averaging over the random vibrational and rotational degrees of freedom of the molecule, could be expressed as follows $[6,16,17]$ (atomic units $\hbar=e=m_{e}=1$ are used throughout the 
work, unless otherwise noted):

$$
\begin{aligned}
\frac{\mathrm{d} \sigma_{\mathrm{el}}^{\mathrm{IAM}}}{\mathrm{d} \Omega}= & \left\langle|F(\theta, k)|^{2}+|G(\theta, k)|^{2}\right\rangle \\
= & \sum_{m=1}^{N} \sum_{n=1}^{N}\left[f_{m}(\theta, k) f_{n}^{*}(\theta, k)+g_{m}(\theta, k) g_{n}^{*}(\theta, k)\right] \\
& \times \frac{\sin \left(s r_{n m}\right)}{s r_{n m}} .
\end{aligned}
$$

Here $\theta$ is the scattering angle; $f_{m}$ and $g_{m}$ are the direct and spin-flip scattering amplitudes of the $m$-th atom, respectively; $s=2 k \sin (\theta / 2)$ and $k=\sqrt{2 E}$, where $E$ is the energy of the incident electron; $r_{n m}$ is the internuclear distance between the $m$-th and $n$-th atom of the molecule.

On the other hand, according to the "Additivity Rule" (IAM-AR) approximation, the DCS (3) could be expressed as the sum of the DCSs of scattering on all particular atoms, i.e. $\sum_{m=1}^{N} \mathrm{~d} \sigma_{\mathrm{el}, m} / \mathrm{d} \Omega=\mathrm{d} \sigma_{\mathrm{el}}^{\mathrm{IAM}}-\mathrm{AR} / \mathrm{d} \Omega$, and of an interference (indirect term):

$$
\begin{aligned}
\frac{\mathrm{d} \sigma_{\mathrm{el}}^{\mathrm{IAM}}}{\mathrm{d} \Omega}= & \frac{\mathrm{d} \sigma_{\mathrm{el}}^{\mathrm{IAM}-\mathrm{AR}}}{\mathrm{d} \Omega}+\sum_{m, n \neq m}\left[f_{m}(\theta, k) f_{n}^{*}(\theta, k)\right. \\
& \left.+g_{m}(\theta, k) g_{n}^{*}(\theta, k)\right] \cdot \frac{\sin \left(s r_{n m}\right)}{s r_{n m}} .
\end{aligned}
$$

The DCSs of electron scattering by an $\mathrm{XY}_{n}$ heteronuclear molecule have a complex character in the IAM framework. For example, in case of scattering by the $\mathrm{CF}_{4}$ molecule, for which all internuclear distances between the $\mathrm{C}$ and $\mathrm{F}$ atoms are equal and very close to the $\mathrm{F}-\mathrm{F}$ distances, the DCS could be calculated as follows:

$$
\begin{aligned}
\frac{\mathrm{d} \sigma_{\mathrm{el}}^{\mathrm{IAM}-\mathrm{AR}}}{\mathrm{d} \Omega}= & \frac{\mathrm{d} \sigma_{\mathrm{el}, \mathrm{C}}}{\mathrm{d} \Omega}+4 \frac{\mathrm{d} \sigma_{\mathrm{el}, \mathrm{F}}}{\mathrm{d} \Omega}, \\
\frac{\mathrm{d} \sigma_{\mathrm{el}}^{\mathrm{IAM}}}{\mathrm{d} \Omega}= & \frac{\mathrm{d} \sigma_{\mathrm{el}, \mathrm{C}}}{\mathrm{d} \Omega}+4 \frac{\mathrm{d} \sigma_{\mathrm{el}, \mathrm{F}}}{\mathrm{d} \Omega}\left[1+3 \frac{\sin \left(s r_{\mathrm{FF}}\right)}{s r_{\mathrm{FF}}}\right] \\
& +4\left[f_{\mathrm{C}} f_{\mathrm{F}}^{*}+f_{\mathrm{F}} f_{\mathrm{C}}^{*}+g_{\mathrm{C}} g_{\mathrm{F}}^{*}+g_{\mathrm{F}} g_{\mathrm{C}}^{*}\right] \frac{\sin \left(s r_{\mathrm{CF}}\right)}{s r_{\mathrm{CF}}} .
\end{aligned}
$$

As one can see in equations (5) and (6), the features and the behaviour of the electron-molecule DCSs in the IAM framework are most likely determined by the energy and angular behaviour of the particular atomic DCSs - $\mathrm{d} \sigma_{\text {el, }} / \mathrm{d} \Omega$ (in our case these are the $\mathrm{d} \sigma_{\text {el, C }} / \mathrm{d} \Omega$ and $\mathrm{d} \sigma_{\mathrm{el}, \mathrm{F}} / \mathrm{d} \Omega$ atomic cross sections).

The integral elastic scattering cross sections could be calculated by direct integration of the corresponding DCSs over the scattering angles:

$$
\begin{gathered}
\sigma_{\mathrm{el}}^{\mathrm{IAM}}(E)=2 \pi \int_{0}^{\pi} \mathrm{d} \theta \sin \theta \frac{\mathrm{d} \sigma_{\mathrm{el}}^{\mathrm{IAM}}(E, \theta)}{\mathrm{d} \theta}, \\
\sigma_{\mathrm{el}}^{\mathrm{IAM}-\mathrm{AR}}(E)=2 \pi \int_{0}^{\pi} \mathrm{d} \theta \sin \theta \frac{\mathrm{d} \sigma_{\mathrm{el}}^{\mathrm{IAM}-\mathrm{AR}}(E, \theta)}{\mathrm{d} \theta} .
\end{gathered}
$$

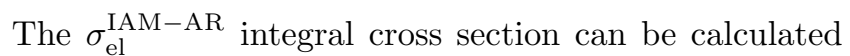
according to the optical theorem as well $[16,17,27]$. This theorem coincides with the IAM-AR approximation $[6,17-$ 19]. Therefore, according to equations (7) and (8) and that $\sin \left(s r_{n m}\right) /\left.s r_{n m}\right|_{\theta \rightarrow 0} \rightarrow 1, \sin \left(s r_{n m}\right) /\left.s r_{n m}\right|_{r_{n m} \rightarrow 0} \rightarrow 1$, the following expression could be derived:

$$
\begin{aligned}
\sigma_{\mathrm{el}}^{\mathrm{IAM}-\mathrm{AR}}(E) & =\frac{4 \pi}{k} \sum_{n=1}^{N} \operatorname{Im}\left[f_{n}(\theta=0, k)\right] \\
& =\sum_{n=1}^{N} \sigma_{\mathrm{el}, n}(E) .
\end{aligned}
$$

The spin-flip amplitude does not contribute to the cross sections at $\theta=0^{\circ}$ at all, so $g_{n}\left(\theta=0^{\circ}, k\right)=0$.

The corresponding $\sigma_{\text {mom }}^{\mathrm{IAM}}-\mathrm{AR}$ and $\sigma_{\text {mom }}^{\mathrm{IAM}}$ momentumtransfer cross sections could be determined analogously, using the $(1-\cos \theta)$ weighting function (see [28]), e.g.:

$$
\begin{aligned}
\sigma_{\mathrm{mom}}^{\mathrm{IAM}-\mathrm{AR}}(E)= & 2 \pi \int_{0}^{\pi} \mathrm{d} \theta \sin \theta(1-\cos \theta) \\
& \times \frac{\mathrm{d} \sigma_{\mathrm{mom}}^{\mathrm{IAM}-\mathrm{AR}}(E, \theta)}{\mathrm{d} \theta} .
\end{aligned}
$$

Based on our previous experience [24-26], we suppose that the scattering cross sections for the whole molecule can be described well, when using a sufficiently good theoretical description of scattering by the particular atoms of the molecule, so not only in case of fast incident electrons, when $k\left(r_{n m}\right)_{\min } \gg 1$, but also at lower energies, in case of $k\left(r_{n m}\right)_{\min }>1$.

The electron-atom scattering amplitudes can be calculated by determining the real $\delta_{\ell}^{ \pm}(E)=\varepsilon_{\ell}^{ \pm}(E)$ (in case of real interaction potential [23]) or the complex $\delta_{\ell}^{ \pm}(E)=$ $\varepsilon_{\ell}^{ \pm}(E)+i \mu_{\ell}^{ \pm}(E)$ partial phase shifts [29] (in case of complex OP, by taking into account the absorption effects). Using real partial phase shifts the scattering amplitudes can be calculated as follows:

$$
\begin{aligned}
f_{m}(\theta, E)= & \frac{1}{2 i k} \sum_{\ell=0}^{\infty}\left[(\ell+1)\left[\exp \left(2 i \varepsilon_{\ell}^{+}\right)-1\right]\right. \\
& \left.+\ell\left[\exp \left(2 i \varepsilon_{\ell}^{-}\right)-1\right]\right] P_{\ell}(\cos (\theta))
\end{aligned}
$$

$$
\begin{aligned}
g_{m}(\theta, E)= & \frac{1}{2 i k} \sum_{\ell=1}^{\infty}\left[\exp \left(2 i \varepsilon_{\ell}^{-}\right)-\exp \left(2 i \varepsilon_{\ell}^{+}\right)\right] \\
& \times P_{\ell}^{1}(\cos (\theta))
\end{aligned}
$$

while using complex partial phase shifts for calculations the scattering amplitudes are:

$$
\begin{aligned}
f_{m}(\theta, E)= & \frac{1}{2 i k} \sum_{\ell=0}^{\infty}(\ell+1)\left[\frac{\exp \left(2 i \varepsilon_{\ell}^{+}\right)}{\exp \left(2 \mu_{\ell}^{+}\right)}-1\right] \\
& +\ell\left[\frac{\exp \left(2 i \varepsilon_{\ell}^{-}\right)}{\exp \left(2 \mu_{\ell}^{-}\right)}-1\right] P_{\ell}(\cos (\theta)) \\
g_{m}(\theta, E)= & \frac{1}{2 i k} \sum_{\ell=1}^{\infty}\left[\frac{\exp \left(2 i \varepsilon_{\ell}^{-}\right)}{\exp \left(2 \mu_{\ell}^{-}\right)}-\frac{\exp \left(2 i \varepsilon_{\ell}^{+}\right)}{\exp \left(2 \mu_{\ell}^{+}\right)}\right] \\
& \times P_{\ell}^{1}(\cos (\theta)) .
\end{aligned}
$$


In equations (11)-(14) $P_{\ell}(\cos (\theta)$ are the Legendre polynomials, while $P_{\ell}^{1}(\cos (\theta)$ are the first order associated Legendre functions.

At the initial $\ell \leq \ell_{\min }$ angular momenta values for the incident electron the partial phase shifts could be determined by the variable-phase method, using the real or complex OP approach (see $[23,28]$ and Refs. therein). The asymptotic values of the phase shifts at $\ell_{\min }<\ell<\ell_{\max }$ are calculated as follows:

$$
\tan \delta_{\ell}^{\mathrm{as}}=\pi \alpha_{\mathrm{d}}(0) k^{2} /[(2 \ell+3)(2 \ell+1)(2 \ell-1)],
$$

where $\alpha_{\mathrm{d}}$ is the static dipole polarizability of the corresponding atom. It can be calculated by any timedependent $a b$ initio quantum approaches, and its empirical value could be also used (see $[30,31])$. For example, at $50 \mathrm{eV}$ for the carbon and fluorine atoms $l_{\min }(\mathrm{C})=13$ and $l_{\min }(\mathrm{F})=11$, while at $1000 \mathrm{eV}$ collision energy these values equal 40 and 34 , respectively. The $l_{\max }$ values were not larger than 295, and they have changed with the collision energy.

It is worth noting here that any published partial phase shift data for electron-atom scattering could be used in the IAM framework to calculate the different cross sections of electron scattering by those molecules, which consist of these atoms.

\subsection{Electron-atom interaction potentials}

In the Relativistic-Static-Exchange-Polarization (RSEP) approximation our electron-atom interaction potential does not contain any empirical or fitting parameters [23]:

$$
\begin{aligned}
V^{ \pm}(r, E)= & V_{\mathrm{S}}(r)+V_{\mathrm{E}}(r, E)+V_{\mathrm{P}}(r) \\
& +V_{\mathrm{R}}(r, E)+V_{\mathrm{SO}}^{ \pm}(r, E),
\end{aligned}
$$

where the " \pm " sign in the spin-orbit interaction potential corresponds to the $j=\ell \pm 1 / 2$ total angular momenta of the incident electron. The $V_{\mathrm{S}}, V_{\mathrm{E}}, V_{\mathrm{P}}, V_{\mathrm{R}}$ and $V_{\mathrm{SO}}^{ \pm}$parts of the OP are the static, exchange, polarization, scalarrelativistic and spin-orbit interaction potentials, respectively. These components are basically determined by the total and spin electron densities of the particular atoms of the molecule. The electron densities could be calculated by different theoretical models: Thomas-Fermi, Hartree-Fock, density functional theory (DFT), etc. The calculated densities usually could be approximated by some analytical functions, which is especially useful in systematic calculations. It is worth noting that in references [20] and [21] the interaction potentials are derived from purely molecular electron densities.

The static potential is determined by the Coulomb interaction between the incident electron and the atomic nuclei as well as between the bound electrons of the target atom (with $\rho(\boldsymbol{r})$ electron density) [32,33]:

$$
V_{\mathrm{S}}(r)=-\frac{Z}{r}+\int \mathrm{d} \boldsymbol{r}^{\prime} \frac{\rho\left(\boldsymbol{r}^{\prime}\right)}{\left|\boldsymbol{r}-\boldsymbol{r}^{\prime}\right|} .
$$

We used the Hartree-Fock electron densities and static potentials [32] of the $\mathrm{C}$ and $\mathrm{F}$ atoms, which are the constituents of the investigated molecular targets.

The spin-orbit interaction potential is (see [32]):

$$
V_{\mathrm{SO}}^{ \pm}(r, E)=\zeta^{ \pm}(j, \ell) \frac{\chi}{r} \frac{\mathrm{d} V_{\mathrm{S}}}{\mathrm{d} r},
$$

where $\chi=\alpha^{2} /\left[2+\alpha^{2}\left(E-V_{\mathrm{S}}\right)\right] ; \zeta^{+}(j, \ell)=\ell / 2$ for $j=$ $\ell+1 / 2$ and $\zeta^{-}(j, \ell)=-(\ell+1) / 2$ for $j=\ell-1 / 2$, while $\alpha$ is the fine structure constant.

The scalar part of the $V_{\mathrm{R}}(r, E)$ relativistic potential is expressed as (see $[35,36])$ :

$$
V_{\mathrm{R}}(r, E)=-\frac{\alpha^{2}}{2} V_{\mathrm{S}}^{2}+\frac{\chi}{4} \frac{\mathrm{d}^{2} V_{\mathrm{S}}}{\mathrm{d} r^{2}}+\frac{3 \chi^{2}}{8}\left(\frac{\mathrm{d} V_{\mathrm{S}}}{\mathrm{d} r}\right)^{2} .
$$

As one can see in equations (18) and (19), using analytical expressions for the static potential is very favourable, because its derivatives then could be also calculated analytically.

For the exchange interaction potential the inhomogeneous electron gas approximation is used (see [33]):

$$
V_{\mathrm{E}}(r, E)=-\frac{k_{\mathrm{F}}(r)}{\pi}\left(1+\frac{1-\kappa^{2}}{2 \kappa} \ln \left|\frac{1+\kappa}{1-\kappa}\right|\right),
$$

where $k_{\mathrm{F}}(r)=\left[3 \pi^{2} \rho(r)\right]^{1 / 3}$ is a function of the density, $\kappa(r, E)=k_{s}(r, E) / k_{\mathrm{F}}(r),\left[k_{s}(r, E)\right]^{2}=k^{2}+V\left(r, k^{2} / 2\right)$, $k^{2}=2 E$. For the $V\left(r, k^{2} / 2\right)$ function the $V\left(r, k^{2} / 2\right)=$ $\left[k_{\mathrm{F}}(r)\right]^{2}+2 I /\left[1+(k r)^{2} / 2\right]$ expression was used with atomic ionization potentials $(I)$. It can be treated as a multiplicative factor for the non-relativistic potential (20).

The polarization potential is determined in the local, spin-unpolarized inhomogeneous electron gas approximation (see $[24,28]$ ) and can be divided into short-range (SR) and long-range (LR) parts. A parameter-free electron correlation-polarization interaction potential is used for the $V_{P}^{\mathrm{SR}}$ short-range part (see [23]). In the local density approximation (LDA) of DFT it can be expressed using correlation functionals:

$$
E_{c}^{\mathrm{LDA}}[\rho]=\int \mathrm{d} \boldsymbol{r} \rho(\boldsymbol{r}) \cdot \varepsilon_{c}[\rho(\boldsymbol{r})],
$$

where $\varepsilon_{c}[\rho(\boldsymbol{r})]=\varepsilon_{c}\left[r_{s}(\boldsymbol{r})\right]$ is the correlation energy density, $r_{s}(\boldsymbol{r})=\{3 /[4 \pi \cdot \rho(\boldsymbol{r})]\}^{1 / 3}$ - the Wigner-Seitz radius. Applying the variation principle for equation (21) once the following polarization potential could be obtained:

$$
V_{P}^{\mathrm{SR}}(r)=\varepsilon_{c}\left(r_{s}\right)-\frac{r_{s}}{3} \frac{\mathrm{d} \varepsilon_{c}}{\mathrm{~d} r_{s}}
$$

The polarization potential can be expressed simply using the $\varepsilon_{c}\left[r_{s}(r)\right]$ correlation energy density, like in reference [38], but equation (22) is a more precise form.

At asymptotic distances the polarization potential has a well-known $V_{P}^{\mathrm{LR}}(r)=-\alpha_{\mathrm{d}}(0) / 2 r^{4}$ form, where $\alpha_{\mathrm{d}}$ is the static dipole polarizability of the particular atoms. We used $\alpha_{\mathrm{d}}^{\mathrm{C}}=11.26 \mathrm{a}_{0}^{3}$ and $\alpha_{\mathrm{d}}^{\mathrm{F}}=3.76 \mathrm{a}_{0}^{3}$ values for the carbon and fluorine atoms, respectively. The $V_{P}^{\mathrm{SR}}(r)$ and $V_{P}^{\mathrm{LR}}(r)$ potentials match at a given $r_{c}$ distance. 
The absorption effects in electron-atom collisions are studied in the complex optical potential (RSEPA) approximation, where $V_{\text {opt }}^{ \pm}(r, E)=V^{ \pm}(r, E)+\mathrm{i} V_{\mathrm{A}}^{ \pm}(r, E)$. They have an impact on the scattering characteristics at $E>\Delta$ collision energies, where $\Delta$ is the energy of the first inelastic threshold of the atoms. For the carbon and fluorine atoms the inelastic effects should be taken into account above $\Delta_{\mathrm{C}}=7.50 \mathrm{eV}$ and $\Delta_{\mathrm{F}}=12.70 \mathrm{eV}$ energies [31]. The absorption effects could be determined, for example, by the non-empirical Staszewska-type potential [40] (see also [28]). This potential has the following form:

$$
V \operatorname{af}(r, E)=-\nu_{\text {loc }}(r, E) \cdot \rho(r) \cdot \bar{\sigma}_{b}(r, E) / 2,
$$

where the local velocity of the incident electron is determined from its local kinetic energy: $\nu_{\mathrm{loc}}(r, E)=\left[2 T_{\mathrm{loc}}\right]^{1 / 2}$, $T_{\mathrm{loc}}(r, E)=E-V_{\mathrm{S}}(r)-V_{\mathrm{E}}(r, E)-V_{\mathrm{P}}(r)$. The values of $\bar{\sigma}_{b}(r, E)$ (average binary collision cross sections) depend on the expressions for $\alpha(r, E)$ and $\beta(r, E)$ functions [40]. For example, in the 2nd version of the Staszewska potential (23) they are used with the following parameters: $\alpha(r, E)=k_{\mathrm{F}}^{2}+\Delta-2\left(V_{\mathrm{S}}+V_{\mathrm{E}}\right), \beta(r, E)=\alpha(r, E)$.

For qualitative calculations the empirical McCarthypotential can be a very useful option (see [40]):

$$
V \operatorname{aMc}(r, E)=-W(E) \cdot r^{2} \rho_{\mathrm{H}}(r) /\left[T_{\mathrm{loc}}(r, E)\right]^{2},
$$

where $\rho_{\mathrm{H}}(r)$ is the density of the highest occupied (valence) electron subshell. The energy-dependent $W(E)$ function can be evaluated by fitting the absorption (excitation or ionization) cross sections to the experimental data. The $W(E)$ function can be used then at all collision energies.

In the spherical [20] and single-centre [21] approaches the absorption effects are taken into consideration more accurately, calculating the absorption of the whole molecule. It is widely known that taking into account the absorption effects slightly decreases the calculated values of the differential and integral cross sections.

\subsection{Interatomic distances of the molecules}

The equilibrium internuclear distances of the $\mathrm{CF}_{n}(n=$ 1 - 4) molecules were calculated by ab initio geometry optimization, using the GAUSSIAN quantum chemistry software [41]. The calculations were performed on the $\operatorname{CCSD}(\mathrm{T})$ level of theory, using the "aug-cc-pvdz" basis set. The following internuclear distances were calculated:

- for CF molecule: $r_{\mathrm{CF}}=1.3071 \AA$

- for $\mathrm{CF}_{2}$ molecule: $r_{\mathrm{CF}}=1.3071 \AA, r_{\mathrm{FF}}=2.0922 \AA$;

- for $\mathrm{CF}_{3}$ molecule: $r_{\mathrm{CF}}=1.3365 \AA, r_{\mathrm{FF}}=2.2053 \AA$;

- for $\mathrm{CF}_{4}$ molecule: $r_{\mathrm{CF}}=1.3370 \AA, r_{\mathrm{FF}}=2.1831 \AA$.

As one can see, the $r_{\mathrm{CF}}$ internuclear distances slightly increase as the number of fluorine atoms increases. The $r_{\mathrm{FF}}$ internuclear distances are not so monotonous - they have a maximum at $n=3$ in case of $\mathrm{CF}_{3}$. For the $\mathrm{CF}, \mathrm{CF}_{2}$ and $\mathrm{CF}_{3}$ radicals the following $r_{\mathrm{CF}}$ internuclear distances were found in reference [42] (in $\AA$, respectively): 1.2912, $1.3018,1.3388$. As one can see, our calculated values are in good overall agreement with the mentioned data.

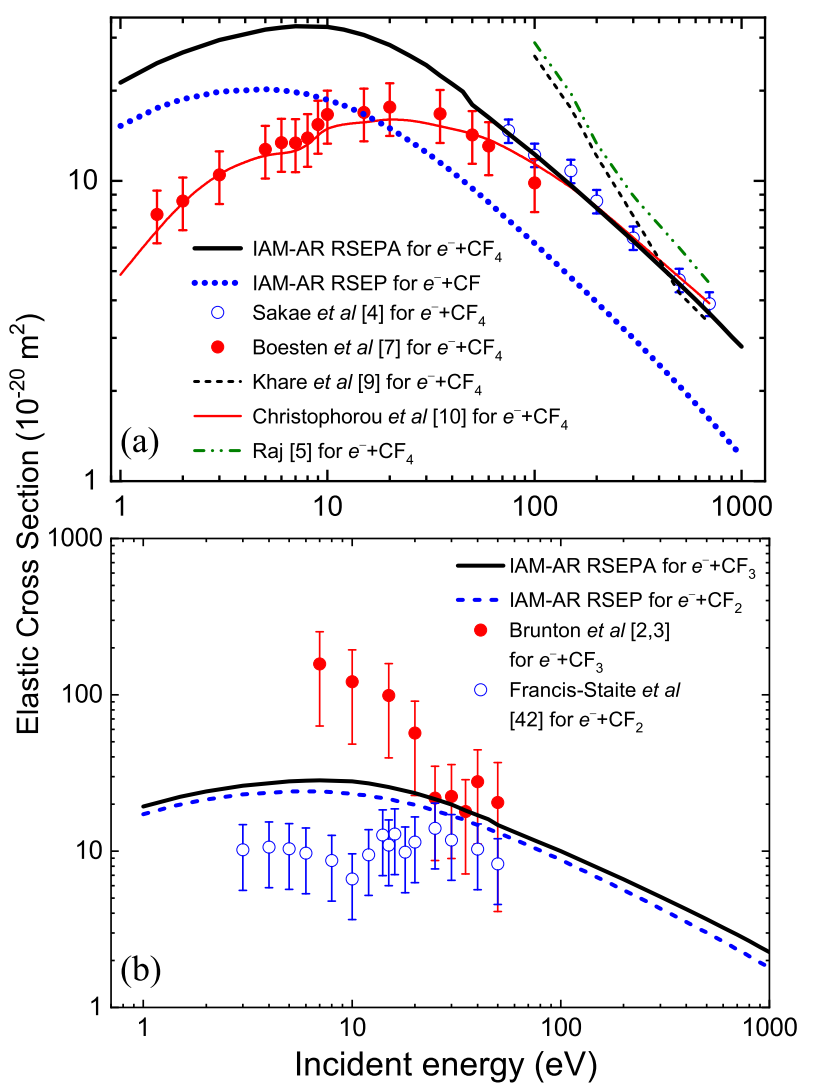

Fig. 1. Elastic integral cross sections of electron scattering by $\mathrm{CF}$ and $\mathrm{CF}_{4}$ (a) as well as by $\mathrm{CF}_{2}$ and $\mathrm{CF}_{3}$ (b) molecules.

\section{Results and discussion}

\subsection{Integral cross sections}

Figures 1 and 2 show the integral elastic and momentumtransfer cross sections, calculated for $e^{-}+\mathrm{CF}_{n}(n=1-4)$ collisions using the IAM-AR approach, which corresponds to the optical theorem (see Eq. (9)). All elastic and momentum-transfer integral cross sections are calculated up to $1000 \mathrm{eV}$ energies. The elastic ICSs for $e^{-}+\mathrm{CF}_{n}$ $(n=1-4)$ scattering are also shown in Table 1 from 10 to $1000 \mathrm{eV}$ collision energies. For $e^{-}+\mathrm{CF}_{3}$ and $e^{-}+\mathrm{CF}_{4}$ collisions the electron-atom scattering amplitudes are calculated in the RSEPA approximation (including the absorption effects), while for the $e^{-}+\mathrm{CF}$ and $e^{-}+\mathrm{CF}_{2}$ collisions we excluded the inelastic effects (RSEP approximation). As we mentioned above, taking into account the absorption effects slightly decreases the absolute values of the cross sections, but it does not affect their qualitative behaviour. We found that the integral cross sections of scattering by the studied molecules can be characterized with very similar energy behaviour.

$e^{-}+\mathbf{C F}$ and $e^{-}+\mathbf{C F}_{4}$. Several experimental data are available for $e^{-}+\mathrm{CF}_{4}$ scattering $[4,7,10]$, however no measurements can be found in the literature for the $e^{-}+$ $\mathrm{CF}$ collision.

Our calculated elastic integral cross sections for $e^{-}+$ $\mathrm{CF}_{4}$ are close to the upper error barrier of experiment [7] 


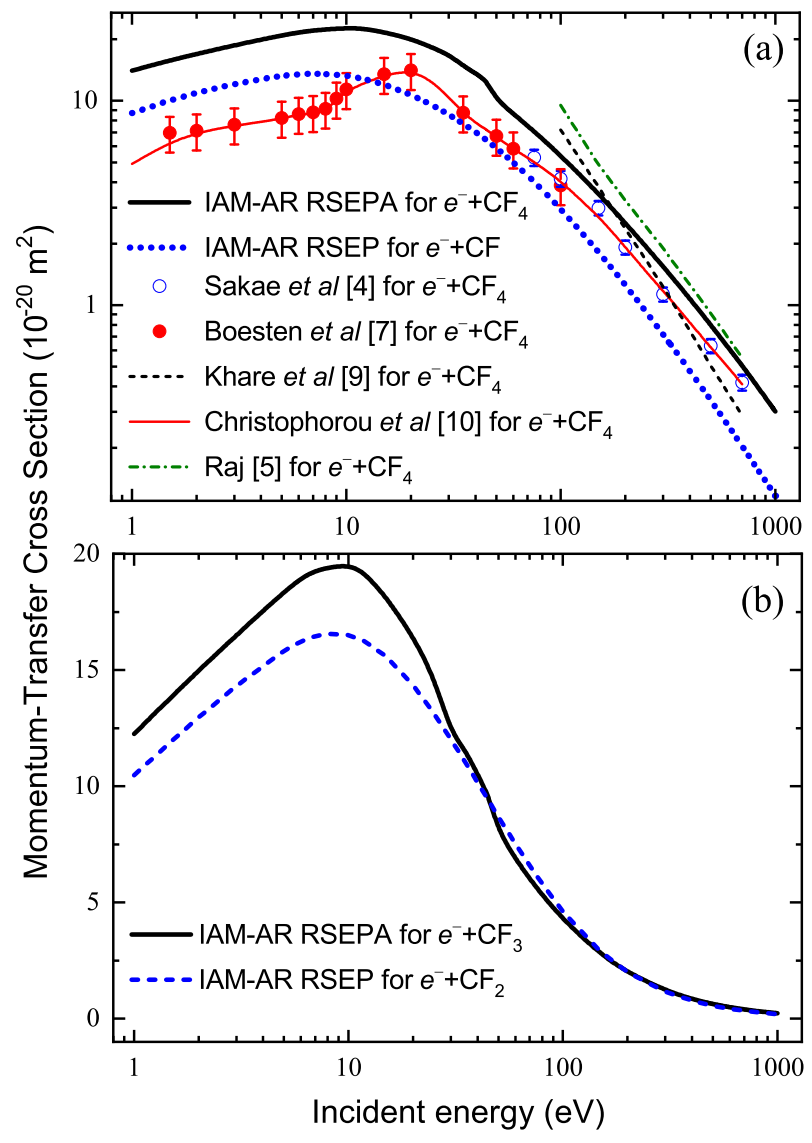

Fig. 2. Momentum-transfer integral cross sections of electron scattering by $\mathrm{CF}$ and $\mathrm{CF}_{4}$ (a) as well as by $\mathrm{CF}_{2}$ and $\mathrm{CF}_{3}$ (b) molecules.

at $50 \mathrm{eV}$. Below this energy we overestimate the data of work [10]. Above $50 \mathrm{eV}$ collision energies we got a good agreement with the data of both references [4] and [10].

For low collision energies, up to $10 \mathrm{eV}$ the main contribution in the calculated ICSs is originated from the cross sections of $e^{-}+\mathrm{C}$ collision, while above $10 \mathrm{eV}-$ from the $e^{-}+\mathrm{F}$ scattering (see the cross section analysis in [26]). The significant overestimation of our calculated cross sections compared to the experimental ones is due to the fact that the contribution of the carbon atom is substantially smaller than the contribution of the fluorine atoms at this energy. In other words, the carbon atom is screened by the fluorine atoms. As the energy increases, the crosssection amplitudes for both the $\mathrm{C}$ and $\mathrm{F}$ atoms decrease, and above $100 \mathrm{eV}$ they are almost equal. Therefore, the total cross section of $e^{-}+\mathrm{CF}_{4}$ scattering is mainly determined by the contribution of the fluorine atoms.

The momentum-transfer cross sections of this process (Fig. 2a) slightly overestimate the experimental data [4,7], but the overall agreement is very good, there are energy regions, where we slightly overestimate the experiments.

There are published theoretical integral and differential cross sections for $e^{-}+\mathrm{CF}_{4}$ collision [5,9] between 100 and $700 \mathrm{eV}$ energies, calculated by the IAM approach. The elastic ICSs [9] are comparable with our data and the measured ones [4] only at higher energies, above $400 \mathrm{eV}$.
Table 1. Theoretical elastic integral cross sections for the different $e^{-}+\mathrm{CF}_{n}(n=1-4)$ scattering processes.

\begin{tabular}{|c|c|c|c|c|}
\hline $\begin{array}{l}\boldsymbol{E} \\
\mathrm{eV}\end{array}$ & $\begin{array}{l}\sigma_{\text {el, }}^{\mathbf{I A F}} \mathbf{C F} \\
10^{-20} \mathrm{~m}^{2}\end{array}$ & $\begin{array}{l}\sigma_{\mathrm{el}, \mathbf{C F}_{\mathbf{2}}}^{\text {IAM-AR }} \\
10^{-20} \mathrm{~m}^{2}\end{array}$ & $\begin{array}{l}\sigma_{\mathrm{el}, \mathrm{CF}_{\mathbf{3}}}^{\mathrm{IAM}-\mathbf{A R}} \\
10^{-20} \mathrm{~m}^{2}\end{array}$ & $\begin{array}{l}\sigma_{\text {el, }}^{\text {IAM-AF }} \mathbf{C A R} \\
10^{-20} \mathrm{~m}^{2}\end{array}$ \\
\hline 10 & 18.6474 & 23.3225 & 27.9122 & 32.5873 \\
\hline 15 & 16.6957 & 21.5388 & 25.7570 & 30.6002 \\
\hline 20 & 14.9905 & 19.7652 & 23.5469 & 28.3216 \\
\hline 25 & 13.5945 & 18.2049 & 21.5244 & 26.1348 \\
\hline 30 & 12.4532 & 16.8661 & 19.8964 & 24.3093 \\
\hline 35 & 11.5059 & 15.7170 & 18.1925 & 22.4035 \\
\hline 40 & 10.7072 & 14.7253 & 16.9943 & 21.0124 \\
\hline 45 & 10.0253 & 13.8642 & 15.9801 & 19.8190 \\
\hline 50 & 9.43754 & 13.1120 & 14.6886 & 17.9401 \\
\hline 60 & 8.47786 & 11.8640 & 13.2793 & 16.2448 \\
\hline 75 & 7.40808 & 10.4423 & 11.7377 & 14.3858 \\
\hline 100 & 6.19010 & 8.78428 & 9.98560 & 12.2641 \\
\hline 200 & 3.93891 & 5.64194 & 6.59521 & 8.12084 \\
\hline 300 & 2.98850 & 4.30347 & 5.11277 & 6.30252 \\
\hline 400 & 2.43963 & 3.53035 & 4.25104 & 5.24724 \\
\hline 500 & 2.07394 & 3.01453 & 3.67118 & 4.53773 \\
\hline 600 & 1.80953 & 2.64057 & 3.24641 & 4.01787 \\
\hline 700 & 1.60802 & 2.35465 & 2.91812 & 3.61580 \\
\hline 800 & 1.44860 & 2.12764 & 2.65477 & 3.29290 \\
\hline 900 & 1.31897 & 1.94242 & 2.43780 & 3.02655 \\
\hline 1000 & 1.21127 & 1.78801 & 2.25530 & 2.80224 \\
\hline
\end{tabular}

The calculated momentum-transfer cross sections in reference [9] overestimate our cross sections up to $150 \mathrm{eV}$, while at higher energies they are in good agreement with our data and those of experiment [4]. It is worth noting that in reference [9] all components of the OP was used, while the authors of reference [5] used only the static and exchange potentials (SE-approximation). The elastic and momentum-transfer ICSs, calculated in [5], overestimate the cross sections, obtained by the authors of [4] and [9].

$e^{-}+\mathbf{C F}_{2}$ and $e^{-}+\mathbf{C F}_{3}$. In Figure $1 \mathrm{~b}$ our cross sections are compared with the available experimental data for $e^{-}+\mathrm{CF}_{3}[2,3]$ and $e^{-}+\mathrm{CF}_{2}$ [43] processes (see also $[26,44,45])$. It is worth noting that the experimental data were obtained with a rather high uncertainty (see Fig. 1b), which can be related with the issues of $\mathrm{CF}_{3}$ radical production in pyrolysis (as described in Sect. 1).

The effect of an additional fluorine atom leads to a slight increasing of the $e^{-}+\mathrm{CF}_{3}$ cross sections, compared to those of $e^{-}+\mathrm{CF}_{2}$ scattering. As one can see in Figure $1 \mathrm{~b}$ the energy behaviour of the experimental cross sections for $\mathrm{CF}_{2}$ and $\mathrm{CF}_{3}$ molecules is not well-described by the theoretical ICSs below $20 \mathrm{eV}$. Our calculated cross sections for $e^{-}+\mathrm{CF}_{2}$ are higher, compared to the corresponding experimental ones, while in case of $e^{-}+\mathrm{CF}_{3}$ scattering our data are smaller. For the $e^{-}+\mathrm{CF}_{2}$ collision we slightly overestimate the experiments even above $20 \mathrm{eV}$. A possible reason of this could be the neglect of absorption effects in our calculation, which decreases the ICS values. However, as one can see in Figure 1b, as the number of fluorine atoms in the radicals is decreasing, the amplitudes of the experimental and calculated cross sections are also decreasing. Therefore, in case of $e^{-}+\mathrm{CF}_{2}$ scattering the calculated cross sections are mainly determined by the contribution 


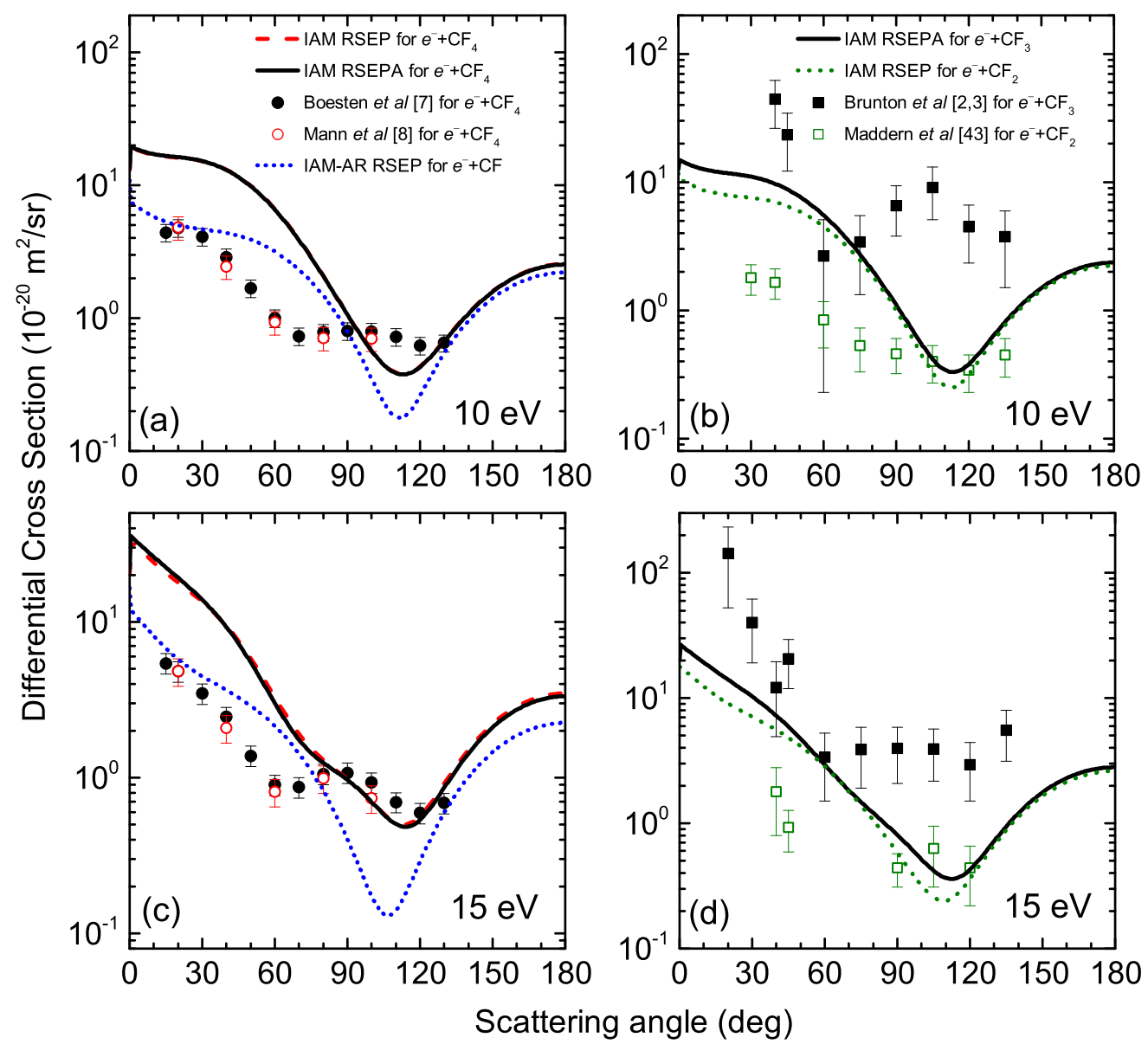

Fig. 3. The angular behaviour of differential cross sections for elastic electron scattering by $\mathrm{CF}_{\text {and }} \mathrm{CF}_{4}(\mathrm{a}, \mathrm{c})$ and also by $\mathrm{CF}_{2}$ and $\mathrm{CF}_{3}(\mathrm{~b}, \mathrm{~d})$ molecules at 10 and $15 \mathrm{eV}$ collision energies.

of the $\mathrm{C}$ atom up to $\sim 35 \mathrm{eV}$ collision energies, while above this - by the contribution of the fluorine atoms (see the $e^{-}+\mathrm{C}$ and $e^{-}+\mathrm{F}$ cross sections in [26]). The overestimation of the calculated cross sections over the experimental ones up to this energy allows one to conclude that the contribution of the carbon atom is again smaller compared to the contribution of the fluorine atoms. The carbon atom somewhat screened by the fluorine atoms, but less effectively than in case of $e^{-}+\mathrm{CF}_{4}$ scattering. As the energy increases, the cross sections for the $\mathrm{C}$ and $\mathrm{F}$ atoms decrease and are closer to each other, so the total cross section for the $\mathrm{CF}_{2}$ radical is mainly determined by the contribution of the fluorine atoms.

The $10-\mathrm{eV}$ minimum of the experimental $e^{-}+\mathrm{CF}_{2}$ cross sections [43] (see Fig. 1b) is not reproduced by our calculations. Our ICSs are quantitatively comparable with the experimental data for $\mathrm{CF}_{2}$ and $\mathrm{CF}_{3}$ molecules above $20 \mathrm{eV}$ collision energies. It is worth noting here that none of the theoretical methods (SMC, IAM-SCAR, $R$-matrix) used in references $[2,3]$ can reproduce the qualitative and quantitative behaviour of the measured cross sections for $e^{-}+$ $\mathrm{CF}_{3}$ scattering. Only the IAM-SCAR method can produce integral cross sections within the experimental error barriers above $25 \mathrm{eV}$, but even these ICSs are smaller than the measured ones. For this particular collision system the experimental cross section is significantly overestimated by our calculations up to $20-25 \mathrm{eV}$ collision energies, which does not coincide with the patterns observed earlier (see [26]). It is possible however, as it was mentioned in reference [26] too, this is an evidence of electron scattering by vibrationally excited $\mathrm{CF}_{3}$ radicals.

Figure $2 \mathrm{~b}$ shows the momentum-transfer integral cross sections. The energy behaviour of these cross sections are similar for all $\mathrm{CF}_{n}$ target molecules. It is worth noting that these ICSs for $e^{-}+\mathrm{CF}_{2}$ and $e^{-}+\mathrm{CF}_{3}$ collisions are almost completely coincide above $\sim 30 \mathrm{eV}$.

\subsection{Differential cross sections}

The angular behaviour of our calculated DCSs are shown in Figures 3-5 for different $e^{-}+\mathrm{CF}_{n}(n=1-4)$ scattering processes. The cross sections are calculated in the IAM framework at 10,15,20,25, 35 and $50 \mathrm{eV}$ collision energies. The scattering amplitudes were calculated for the particular atoms in the RSEP $\left(e^{-}+\mathrm{CF} / \mathrm{CF}_{2}\right)$ and RSEPA $\left(e^{-}+\mathrm{CF}_{3} / \mathrm{CF}_{4}\right)$ approximations of the optical potential model. As we found for the ICSs the inclusion of absorption effects somewhat decreases the absolute DCS values, but does not affect their qualitative behaviour. 


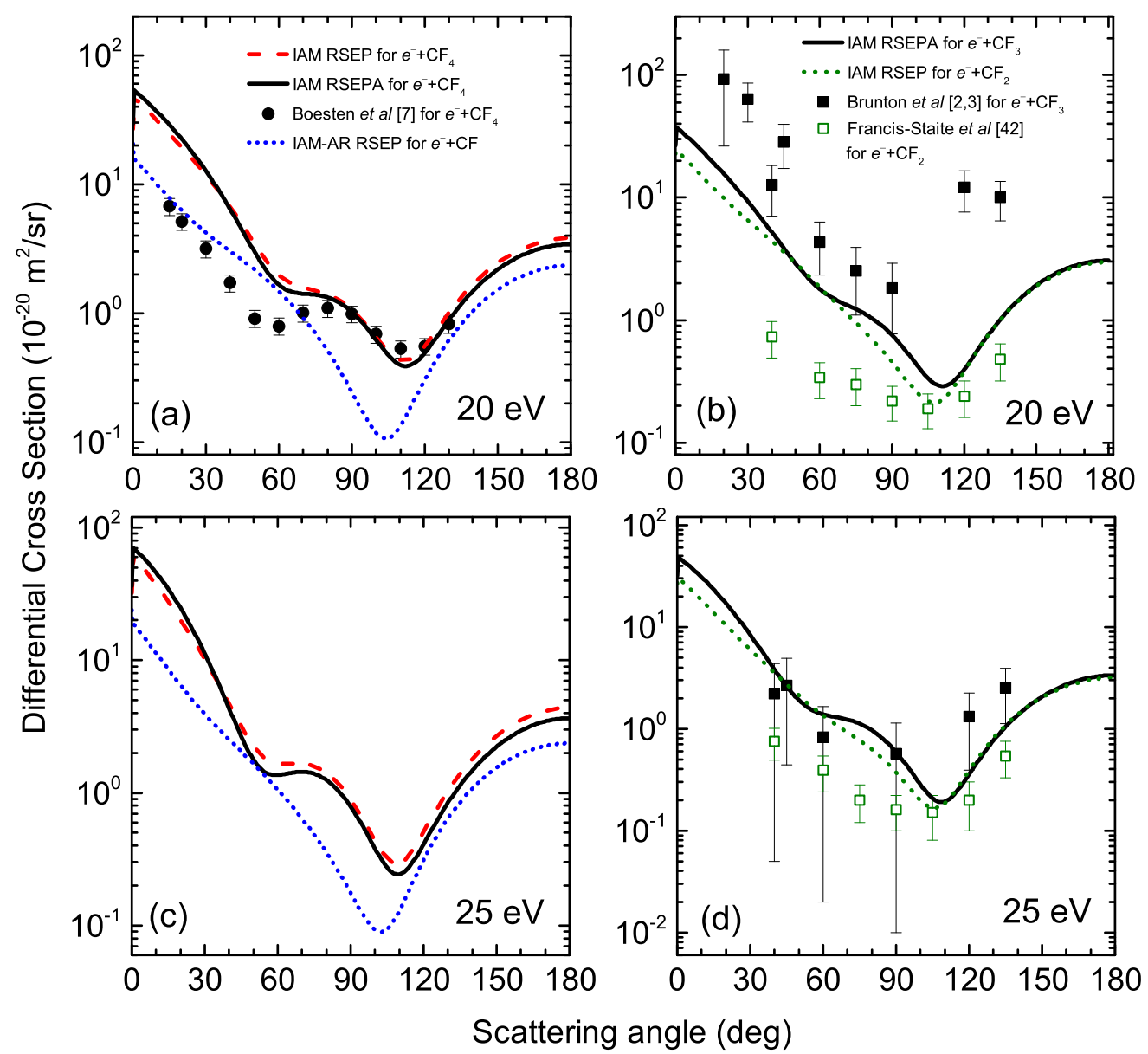

Fig. 4. The angular behaviour of differential cross sections for elastic electron scattering by $\mathrm{CF}_{\text {and }} \mathrm{CF}_{4}(\mathrm{a}, \mathrm{c})$ and also by $\mathrm{CF}_{2}$ and $\mathrm{CF}_{3}(\mathrm{~b}, \mathrm{~d})$ molecules at 20 and $25 \mathrm{eV}$ collision energies.

We found that using the IAM-AR approach for DCS calculations leads to an intensive decreasing of cross section values at small scattering angles, compared with the results of the IAM approach. For example, at $7 \mathrm{eV}$ this angular range is $\left[0^{\circ}-90^{\circ}\right]$. With an increasing collision energy this angular interval substantially decreases, at $50 \mathrm{eV}$ it is about $\left[0^{\circ}-30^{\circ}\right]$. Moreover, equations (3)-(4) include more intensive contributions to the structure of the DCSs. This is due to the role of interference terms in the angular behaviour of the cross sections. The angular features of the calculated DCSs for the mentioned molecules are similar - the absolute value of the cross sections increases step-by-step with the increasing number of fluorine atoms.

In case of the diatomic $\mathrm{CF}$ molecule experimental data could not be found at all in the literature. The angular behaviour of the differential cross sections for the $\mathrm{CF}$ and $\mathrm{CF}_{4}$ molecules are similar, however the DCSs for $e^{-}+\mathrm{CF}_{2}$ and $e^{-}+\mathrm{CF}_{3}$ are much closer to each other. The calculated DCSs by the authors of reference [5] for $e^{-}+\mathrm{CF}_{4}$ scattering overestimate the corresponding experimental [4] and theoretical [9] cross sections at all collision energies (from 100 to $700 \mathrm{eV}$ ) and all scattering angles. The angular behaviour (oscillations) of the theoretical DCSs are typically similar to each other and also to the experimental ones. The results of calculations in reference [9] overestimate the experimental data [4] at 100 and $150 \mathrm{eV}$, but at higher collision energies (200-300 eV) they are in good overall agreement.

In order to calculate the DCSs of $e^{-}+\mathrm{CF}_{x}(x=1-$ 3 ) scattering processes the authors of references [42] and [46] have used the $R$-matrix method below $10 \mathrm{eV}$ collision energies. It is worth noting that our method does not allow to adequately describe quantitatively the differential cross sections at these low energies. For their calculations in the inner region the authors of references $[42,46]$ used the close coupling method with molecular wavefunctions. In the outer region they used the coupled equations of singlecentre expansion. At small scattering angles the DCSs for molecules with large dipole momentum generally characterized with very high values. For example, for the $e^{-}+\mathrm{CF}$ collision the DCS at $7.5 \mathrm{eV}$ and $10^{\circ}$ scattering angle equals $40.6 \times$ $10^{-20} \mathrm{~m}^{2} / \mathrm{sr}$. Our calculated DCS with a $4.42 \times 10^{-20} \mathrm{~m}^{2} / \mathrm{sr}$ value at $7 \mathrm{eV}$ is close to the $3 \times 10^{-20} \mathrm{~m}^{2} / \mathrm{sr}$ theoretical value, which was calculated in reference [42], using small dipole momentum (0.12 Debye). In the $45^{\circ}-130^{\circ}$ angular range the calculated DCSs in [42] have a clear structure - they could be characterized with 3 minima and 2 maxima with ca. $0.5 \times 10^{-20} \mathrm{~m}^{2} / \mathrm{sr}$ and $0.4 \times 10^{-20} \mathrm{~m}^{2} / \mathrm{sr}$ values. Our cross sections for this molecule are characterized with only one wide gap at all collision energies. 


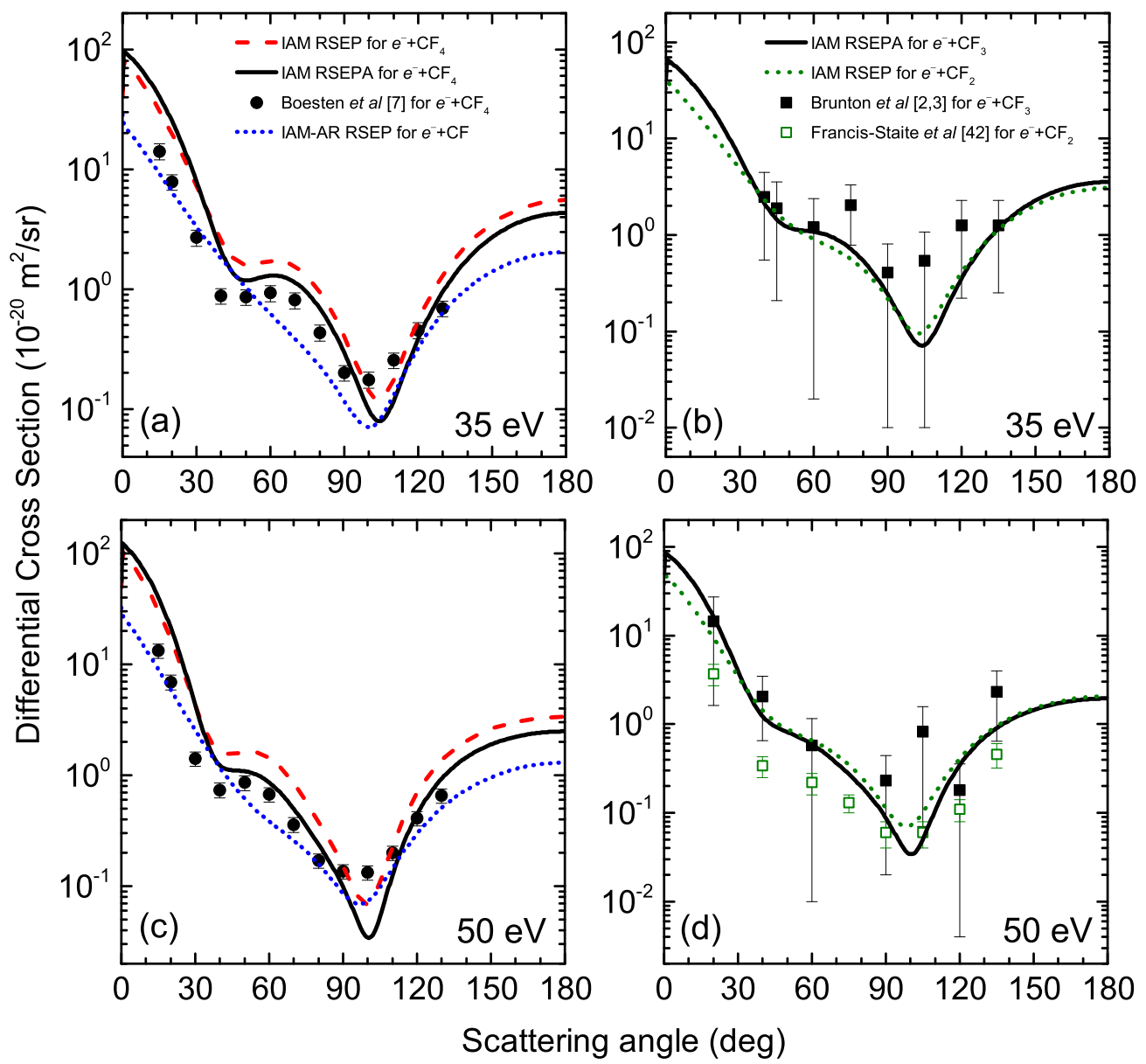

Fig. 5. The angular behaviour of differential cross sections for elastic electron scattering by $\mathrm{CF}_{\text {and }} \mathrm{CF}_{4}(\mathrm{a}, \mathrm{c})$ and also by $\mathrm{CF}_{2}$ and $\mathrm{CF}_{3}(\mathrm{~b}, \mathrm{~d})$ molecules at 35 and $50 \mathrm{eV}$ collision energies.

$10 \mathrm{eV}$. The theoretical cross sections for $e^{-}+\mathrm{CF}$ and $e^{-}+\mathrm{CF}_{4}$ collisions are similar at this energy. The highest difference between them could be observed at small scattering angles, up to $70^{\circ}$. Near the minimum at $110^{\circ}$ the calculated DCS for $e^{-}+\mathrm{CF}_{4}$ is higher than for the $e^{-}+\mathrm{CF}$ collision. We obtained not so good agreement with the angular dependencies, compared to the experimental data $[7,8]$ for this energy. The measured cross sections [7] somewhat overestimate our results near the minima, where the DCSs for the two processes are similar.

Our cross sections for $e^{-}+\mathrm{CF}_{3}$ and $e^{-}+\mathrm{CF}_{2}$ are very similar, they differ only at forward angles and in the region of the minimum. In the $120^{\circ}-180^{\circ}$ angular range they coincide. Our DCSs are close to the measured data $[2,3]$ from $40^{\circ}$ up to $75^{\circ}$. These experimental cross sections have a strong angular structure at this energy - a minimum at $60^{\circ}$ and a maximum at $115^{\circ}$, which is not reproduced by any of the theoretical calculations. The angular behaviour of the calculated $e^{-}+\mathrm{CF}_{2}$ cross sections is closer to the experimental data in reference [44], however it strongly overestimates them below $90^{\circ}$. Near the minimum at $115^{\circ}$ these cross sections are in good agreement with each other and also with the calculated cross sections for the $e^{-}+\mathrm{CF}_{3}$ collision.
$15 \mathrm{eV}$. The angular dependencies of the calculated cross sections for $e^{-}+\mathrm{CF}$ and $e^{-}+\mathrm{CF}_{4}$ collisions already strongly differ at this collision energy. The angular structure of the $e^{-}+\mathrm{CF}_{4}$ DCSs is more complex - another minimum is formed around $70^{\circ}$. Our data are well comparable with the measured ones $[7,8]$ above $80^{\circ}$. The absorption effects are still negligible.

The calculated $e^{-}+\mathrm{CF}_{3}$ and $e^{-}+\mathrm{CF}_{2}$ cross sections are in good agreement, they are almost equal in backward directions, above $125^{\circ}$. At $30^{\circ}-70^{\circ}$ scattering angles our cross sections for the $e^{-}+\mathrm{CF}_{3}$ collision are close to the lower error bar of the experimental data [2,3]. These experimental DCSs could be characterized with an almost stationary ca. $4 \times 10^{-20} \mathrm{~m}^{2} / \mathrm{sr}$ value between $60^{\circ}$ and $135^{\circ}$. This is not reproduced by any theoretical cross sections. The calculated $e^{-}+\mathrm{CF}_{2}$ cross sections slightly overestimate the measured data, obtained by the authors of reference [44] at $40^{\circ}-45^{\circ}$ scattering angles. We also found that the corresponding cross sections are close to each other near the minimum between $90^{\circ}$ and $120^{\circ}$.

$20 \mathrm{eV}$. A higher discrepancy is observed between the calculated differential cross sections of electron scattering by $\mathrm{CF}$ and $\mathrm{CF}_{4}$ molecules. The $e^{-}+\mathrm{CF}$ DCSs preserve their previous structure with a single minimum. However, 
the cross sections for $e^{-}+\mathrm{CF}_{4}$ collision have a more interesting character - a clear formation of another minima is observed at $65^{\circ}$. Our data are in good overall agreement with the experimental ones [7] for this process above $70^{\circ}$. The absorption effects are still negligible.

The calculated $e^{-}+\mathrm{CF}_{3}$ cross sections are also close to the measured ones [2,3] nearly in the whole forward direction (from $20^{\circ}$ to $90^{\circ}$ ). Our DCSs for the $e^{-}+\mathrm{CF}_{2}$ and $e^{-}+\mathrm{CF}_{3}$ collisions are similar, but there are some slight differences between them. For example, in case of the $\mathrm{CF}_{3}$ molecule a second minimum is formed at $60^{\circ}$. In backward direction, from $120^{\circ}$ to $180^{\circ}$, they still coincide. The calculated cross sections for the $e^{-}+\mathrm{CF}_{2}$ collision slightly overestimate the corresponding experimental data [43] at $45^{\circ}-75^{\circ}$ scattering angles, and they are also close to the calculated $\sim 0.2 \times 10^{-20} \mathrm{~m}^{2} / \mathrm{sr}$ value for $e^{-}+\mathrm{CF}_{3}$ scattering near the minimum around $105^{\circ}$.

$25 \mathrm{eV}$. At this collision energy there are no published experimental data for the $e^{-}+\mathrm{CF}_{4}$ scattering process. There is a slightly higher difference between our calculated cross sections for $e^{-}+\mathrm{CF}$ and $e^{-}+\mathrm{CF}_{4}$ scattering. In the $e^{-}+$CF DCSs only a single minimum is observed, as seen for lower energies. The angular dependencies of the $e^{-}+\mathrm{CF}_{4}$ cross sections show a clear additional minimum at $60^{\circ}$. The absorption effects are already noticeable at this energy.

Our calculated cross sections for $e^{-}+\mathrm{CF}_{3}$ scattering are close to the measured ones $[2,3]$, reproducing their features in the whole measured angular range from $40^{\circ}$ up to $135^{\circ}$. The $e^{-}+\mathrm{CF}_{2}$ and $e^{-}+\mathrm{CF}_{3}$ DCSs are still close to each other, but there is a slightly higher difference between their absolute values. They are in a perfect agreement above $110^{\circ}$. The calculated cross sections for $e^{-}+\mathrm{CF}_{2}$ scattering are close to the experimental ones from reference [43] for all measured angles, from $40^{\circ}$ to $135^{\circ}$. However, they slightly overestimate the corresponding experimental data at angles below $90^{\circ}$. In the region of the minimum around $105^{\circ}$ the calculated DCSs for the $\mathrm{CF}_{2}$ and $\mathrm{CF}_{3}$ radicals are nearly equal $\left(\sim 0.2 \times 10^{-20} \mathrm{~m}^{2} / \mathrm{sr}\right)$.

$35 \mathrm{eV}$. Slightly higher discrepancies were observed between the $e^{-}+\mathrm{CF}$ and $e^{-}+\mathrm{CF}_{4}$ differential cross sections at this energy. The single minimum in the $e^{-}+\mathrm{CF}$ DCS is shifted to smaller angles, now it can be found around $100^{\circ}$. Our cross sections for the $e^{-}+\mathrm{CF}_{4}$ collision are in a good qualitative and in satisfactory quantitative agreement with the experimental data, published by the authors of reference [7]. An additional minimum is located at ca. $45^{\circ}$ scattering angle. The absorption effects have a clear impact on the cross section values, which could be observed already in the full angular range.

Our DCSs for the $e^{-}+\mathrm{CF}_{3}$ scattering process reproduce the angular structure of the measured cross sections in $[2,3]$ and close to them in the absolute scale for all investigated scattering angles, from $40^{\circ}$ up to $135^{\circ}$. The calculated $e^{-}+\mathrm{CF}_{2}$ and $e^{-}+\mathrm{CF}_{3}$ cross sections are very similar, only a slight difference can be found between them at small forward angles.

$50 \mathrm{eV}$. At this collision energy a good overall agreement (both qualitative and quantitative) is obtained between our calculated DCSs for the $e^{-}+\mathrm{CF}_{4}$ collision and the corresponding experimental ones [7]. This is valid for all scattering angles, except of the minimum at $100^{\circ}$. Our theoretical DCS is ca. $0.03 \times 10^{-20} \mathrm{~m}^{2} / \mathrm{sr}$ here, while the experimental value is ca. $0.14 \times 10^{-20} \mathrm{~m}^{2} / \mathrm{sr}$. The absorption effects are rather strong here: they reduce the absolute values of the cross sections approximately by a factor of 2 in a wide angular range above $\sim 35^{\circ}$.

The calculated $e^{-}+\mathrm{CF}_{3}$ cross sections are within the estimated uncertainty of the measured data in $[2,3]$. They are close to each other for all scattering angles between $20^{\circ}$ and $135^{\circ}$. At very small angles, below $30^{\circ}$, our DCSs for $e^{-}+\mathrm{CF}_{2}$ and $e^{-}+\mathrm{CF}_{3}$ scattering slightly differ, but at higher angles they are close to each other. The calculated cross sections for the $e^{-}+\mathrm{CF}_{2}$ collision are similar to the measured ones [43] for all investigated angles. Around the minimum at ca. $105^{\circ}$ they slightly overestimate the corresponding experimental data, and close to our DCSs for $e^{-}+\mathrm{CF}_{3}$ scattering. At this energy the absorption effect plays an important role, so using the RSEPA approximation instead of RSEP leads to a better agreement between our calculated data for $\mathrm{CF}_{2}$ and the corresponding experimental ones [43].

To summarize we can say that the DCSs for the $e^{-}+$ $\mathrm{CF}_{4}$ scattering process are in good agreement with the experimental data published in reference [7] above $20 \mathrm{eV}$ collision energies, especially in backward scattering directions. Once the energy increases the agreement gets better, even at small angles, in forward directions. With an increasing collision energy the absorption effects are also increasing - the values of our cross sections are considerably reduced due to the absorption and they are closer to the measured data.

The theoretical $e^{-}+\mathrm{CF}_{3}$ differential cross sections quantitatively can be comparable with the experimental ones at small angles above above $15 \mathrm{eV}$ collision energies. The theoretical DCSs for $e^{-}+\mathrm{CF}_{2}$ scattering reproduce the measured cross sections in backward directions $\left(90^{\circ}-130^{\circ}\right)$ above $10 \mathrm{eV}$ collision energies. Therefore, in case of smaller molecular targets a better agreement can be observed between our theoretical data and the corresponding experimental DCSs at low collision energies.

It is worth noting that the only theory in references $[2,3]$, which qualitatively reproduce the experimental angular behaviour of the DCSs for $e^{-}+\mathrm{CF}_{3}$ scattering, is the Schwinger multichannel method. But even this method underestimates the measured cross sections at $7 \mathrm{eV}$ by an order of magnitude and at least with a factor of 5 at $20 \mathrm{eV}$. Respectively, none of the theoretical methods, proposed in $[2,3]$, can correctly reproduce the absolute values and behaviour of the measured cross sections. The results of IAM-SCAR calculations [2,3] (which are similar to our IAM-AR approximation) are in good overall agreement with the measured elastic scattering data above $25 \mathrm{eV}$ collision energies. These cross sections coincide with the lower boundary values of the experimental error bars.

\section{Conclusions}

In order to study the elastic scattering of electrons by molecular targets the independent atom model is used 
along with parameter-free real and complex electron-atom interaction potentials. The features of electron-molecule scattering generally follow the features of the scattering by its particular atoms. The integral cross sections of electron scattering by the $\mathrm{CF}, \mathrm{CF}_{2}, \mathrm{CF}_{3}$ and $\mathrm{CF}_{4}$ molecular targets are calculated in the IAM-AR approach, while for the differential cross sections the IAM approach is used.

The comparative analysis of our integral cross sections with the available experimental ones shows that our data can be used for the description of scattering by $\mathrm{CF}_{2}$ radicals above $10 \mathrm{eV}$, by $\mathrm{CF}_{3}-$ above $15-20 \mathrm{eV}$, while in case of the $\mathrm{CF}_{4}$ target molecule - above $40 \mathrm{eV}$. For the previous a good agreement was observed between the momentumtransfer cross sections above $50 \mathrm{eV}$ collision energy.

Comparing our theoretical differential cross sections with the corresponding measurements allows one to draw some conclusions about the limits of our methods. They could be used to adequately calculate the DCSs above $10 \mathrm{eV}$ for $\mathrm{CF}_{2}$ (between $100^{\circ}$ and $180^{\circ}$ scattering angles), above $15-20 \mathrm{eV}$ for $\mathrm{CF}_{3}\left(0^{\circ}-90^{\circ}\right)$ and above $20 \mathrm{eV}$ for the largest $\mathrm{CF}_{4}$ molecule (from $80^{\circ}$ to $180^{\circ}$ ).

Comparing our results of calculations with the experimental data for $e^{-}+\mathrm{CF}_{2}$ and $e^{-}+\mathrm{CF}_{3}$ collisions allows one to conclude that in case of the $\mathrm{CF}_{3}$ radical in references $[2,3]$ the scattering characteristics were most likely measured for vibrationally excited target molecules.

The performed calculations and their comparison with the available experiments confirm that more sophisticated methods are needed to develop in order to adequately describe the cross sections of scattering at lower energies. These methods, along with the electron-molecule interaction potentials, should take fully into account the characteristics of the targets - molecular wavefunctions, electron densities, polarizabilities and dipole momenta.

Open access funding provided by MTA Institute for Nuclear Research (MTA ATOMKI). This work was supported by the K128621 and FK132989 NRDIO-OTKA grants. We acknowledge NIIF for awarding us HPC access to resources based in Hungary at Debrecen. The authors are grateful to J. Zs. Mezei for the fruitful discussions.

\section{Author contribution statement}

E.R. and V.K. designed the model and the computational framework for electron-atom scattering theory and performed the calculations of electron-atom scattering amplitudes. S.D. and E.R. carried out the implementation of the model for electron-molecule collisions. S.D. and E.R. performed the cross section calculations in the IAM framework and analysed the data. E.R. conceived the study and were in charge of the overall direction and planning. S.D. and E.R. wrote the manuscript with continuous discussion and support of V.K.

Open Access This is an open access article distributed under the terms of the Creative Commons Attribution License (https://creativecommons.org/licenses/by/4.0/), which permits unrestricted use, distribution, and reproduction in any medium, provided the original work is properly cited.

\section{References}

1. J.S. Yoon, M.Y. Song, H. Kato, M. Hoshino, H. Tanaka, M.J. Brunger, S.J. Buckman, H. Cho, J. Phys. Chem. Ref. Data 39, 033106 (2010)

2. J.R. Brunton, L.R. Hargreaves, S.J. Buckman, G. Garíca, F. Blanco, O. Zatsarinny, K. Bartschat, M.J. Brunger, Chem. Phys. Lett. 568-569, 55 (2013)

3. J.R. Brunton, L.R. Hargreaves, T.M. Maddern, S.J. Buckman, G. García, F. Blanco, O. Zatsarinny, K. Bartschat, D.B. Jones, G.B. da Silva, M.J. Brunger, J. Phys. B: At. Mol. Opt. Phys. 46, 245203 (2013)

4. T. Sakae, S. Sumiyoshi, E. Murakami, Y. Matsumoto, K. Ishibashi, A. Katase, J. Phys. B: At. Mol. Opt. Phys. 22, 1385 (1989)

5. D. Raj, J. Phys. B: At. Mol. Opt. Phys. 24, L431 (1991)

6. D. Raj, Phys. Lett. A 160, 571 (1991)

7. L. Boesten, H. Tanaka, A. Kobayashi, M.A. Dillon, M. Kimura, J. Phys. B: At. Mol. Opt. Phys. 25, 1607 (1992)

8. A. Mann, F. Linder, J. Phys. B: At. Mol. Opt. Phys. 25, $533(1992)$

9. S.P. Khare, D. Raj, P. Sinha, J. Phys. B: At. Mol. Opt. Phys. 27, 2569 (1994)

10. L.G. Christophorou, J.K. Olthoff, M.V.V.S. Rao, J. Phys. Chem. Ref. Data 25, 1341 (1996)

11. L.G. Christophorou, J.K. Olthoff, J. Phys. Chem. Ref. Data 30, 449 (2001)

12. I. Iga, I.P. Sanches, P. Rawat, M.G.P. Homem, M.T. Lee, J. Phys. B: At. Mol. Opt. Phys. 38, 3477 (2005)

13. P. Verma, B. Antony, J. Electron. Spectrosc. Relat. Phenom. 210, 30 (2016)

14. Cz. Szmytkowski, P. Mozejko, Uzhhorod Univ. Sci. Herald. Ser. Phys. 8, 28 (2000)

15. P. Mozejko, B. Zywicka-Mozejko, Cz. Szmytkowski, Uzhhorod Univ. Sci. Herald. Ser. Phys. 8, 108 (2000)

16. N.F. Mott, H.S.W. Massey, The Theory of Atomic Collisions, 3rd ed., corr. reprint (Clarendon Press, Oxford, 1971)

17. P. Mozejko, B. Zywicka-Mozejko, C. Szmytkowski, Nucl. Instrum. Methods Phys. Res. Sect. B 196, 245 (2002)

18. F. Blanco, G. Garcia, Phys. Lett. A 317, 458 (2003)

19. F. Blanco, G. Garcia, Phys. Lett. A 330, 230 (2004)

20. A. Jain, K.L. Baluja, Phys. Rev. A 45, 202 (1992)

21. F.A. Gianturco, J.A. Rodriguez-Ruiz, N. Sanna, Phys. Rev. A 52, 1257 (1995)

22. A.C. Yates, Phys. Rev. 176, 173 (1968)

23. V.I. Kelemen, M.M. Dovhanych, E. Yu. Remeta, Ukr. J. Phys. 59, 569 (2014)

24. Sh. Demesh, E. Remeta, V. Kelemen, in Contributed papers of the 6th Conference on Elementary Processes in Atomic Systems, 2014, edited by S. Matejcik, P. Papp, O. Bogar (Bratislava, Slovakia, 2014), p. 65

25. S.S. Demesh, V.I. Kelemen, E.Y. Remeta, J. Phys. Conf. Ser. 635, $072020(2015)$

26. S.S. Demesh, V.I. Kelemen, E.Y. Remeta, J. Phys. B: At. Mol. Opt. Phys. 50, 135201 (2017)

27. P.G. Burke, Potential Scattering in Atomic Physics (Springer, New York, 2011)

28. V.I. Kelemen, E.Y. Remeta, J. Phys. B: At. Mol. Opt. Phys. 45, 185202 (2012) 
29. E.Y. Remeta, V.I. Kelemen, J. Phys. B: At. Mol. Opt. Phys. 43, 045202 (2010)

30. P. Schwerdtfeger, J.K. Nagle, Mol. Phys. 117, 1200 (2019)

31. A.A. Radsig, B.M. Smirnov, Handbook on Atomic and Molecular physics (Atomizdat, Moscow, 1980)

32. T.G. Strand, R.A. Bonham, J. Chem. Phys. 40, 1686 (1964)

33. V.I. Kelemen, E.Y. Remeta, E.P. Sabad, J. Phys. B: At. Mol. Opt. Phys. 28, 1527 (1995)

34. R.D. Cowan, The Theory of Atomic Structure and Spectra (University of California Press, Berkeley, 1981)

35. V.A. Fock, Fundamentals Of Quantum Mechanics (Mir Publishers, Moscow, 1978)

36. L.T.S.F. Lam, J. Phys. B: At. Mol. Phys. 15, 119 (1982)

37. A.K. Rajagopal, J. Callaway, Phys. Rev. B 7, 1912 (1973)

38. J. K. O'Connell, N.F. Lane, Phys. Rev. A 27, 1893 (1983)

39. N.T. Padial, D.W. Norcross, Phys. Rev. A 29, 1742 (1984)
40. G. Staszewska, D.W. Schwenke, D.G. Truhlar, Phys. Rev. A 29, 3078 (1984)

41. M.J. Frisch, G.W. Trucks, H.B. Schlegel, et al., Gaussian 09, Revision E.01 (Gaussian Inc., Wallingford CT, 2009)

42. I. Rozum, J. Tennyson, J. Phys. B: At. Mol. Opt. Phys. 37, 957 (2004)

43. J.R. Francis-Staite, T.M. Maddern, M.J. Brunger, S.J. Buckman, C. Winstead, V. McKoy, M.A. Bolorizadeh, H. Cho, Phys. Rev. A 79, 052705 (2009)

44. T.M. Maddern, L.R. Hargreaves, J.R. Francis-Staite, M.J. Brunger, S.J. Buckman, C. Winstead, V. McKoy, Phys. Rev. Lett. 100, 063202 (2008).

45. S.J. Buckman, T. Maddern, J. Francis-Staite, L. Hargreaves, M.J. Brunger, G. Garcia, J.C. Lower, S. Mondal, J.P. Sullivan, A. Jones, P. Caradonna, D. Slaughter, C. Mackochekanwa, R.P. McEachran, J. Phys.: Conf. Ser. 133, 012001 (2008)

46. I. Rozum, N.J. Mason, J. Tennyson, J. Phys. B: At. Mol. Opt. Phys. 35, 1583 (2002) 